\title{
COVARIANCE DIFFERENCES OF LINEALY REPRESENTABLE SEQUENCES IN HILBERT SPACES
}

\author{
Muriuki, J.N. \\ Department of Mathematics, College of Biological and Physical Sciences, \\ University of Nairobi P.O. Box 30197-00100, Nairobi
}

\begin{abstract}
TThe paper introduces the concepts of covariance differences of a sequence and establishes its relationship with the covariance function. One of the main results of this paper is the criteria of linear representability of sequences in Hilbert spaces.
\end{abstract}

\section{INTRODUCTION}

In the analysis of stationary random processes and sequences it was observed that there exists a close relationship with the spectral theory of self-adjoint or unitary operators in Hilbert spaces. In the works of Kolmogorov, the theory of stationary random sequences was constructed with the aid of unit parametric group of unitary operators.

In the last years, in the works of Nagia and Foyash [6], Brodskii [7],Livshit [3] and others they developed the theory of nonunitary operators in Hilbert spaces whereby the analogy of spectral theory of nonunitary operators was the triangular and universal models of nonunitary operators. For this reason there arose the problem of studying nonstationary random sequences embedded in Hilbert spaces with the aid of universal models, such that triangular models allows the construction of some elementary nonstationary sequences and with the aid of universal models pick from them more general classes of nonstationary sequences. The paper is devoted to analysing sequences in Hilbert spaces that can be represented in the form $x(n)=T^{n} x_{0}$, where $\mathrm{T}$ is a linear bounded operator. In the terminologies of covariance function we give proof of the necessary and sufficient condition for a possibility of such a representation. The main methods of research are the Kelley's transformations of nonunitary operators that allow the construction of universal models of contraction operators on the basis of universal models of dissipative operators $[3,7,8]$.

\section{EMBEDDING NONSTATIONARY SEQUENCESIN HILBERT SPACE}

Let $\Omega$ be a set of elementary events. If the random function $x(n)=x(n, \omega)$ depends not only on $(\omega \in \Omega)$, but also on $n=0,1,2 \ldots$ then it is called a random sequence.

Let $x(n), \mathrm{n}=0,1,2 \ldots$ Be a random sequence of zero mathematical expectation and a finite second moment:

$M x(n)=0, M|x(n)|^{2}<\infty$

We denote $\widehat{H}$ to be the linear span of all $x(n)$ when $\mathrm{n}$ takes finite natural numbers in $\widehat{H}$

We introduce the scalar product of the elements:

$h=\sum_{k=1}^{n} a_{k} x(k)$ and $g=\sum_{p=1}^{m} b_{p} x(p)_{\text {in the following }}$ way

$(h, g)_{\widehat{H}}=M\left[\sum_{k=1}^{n} a_{k} x(k) \cdot \overline{\sum_{p=1}^{m} b_{p} x(p)}\right]$

On closing we obtain the Hilbert space $H=H_{X}$, embedded with the sequence $x(n)$. Hence any sequence $x(n)$ satisfying the above property can be considered as 
a sequence in Hilbert space $H_{X}$, for which the covariance function is given by the scalar product in $H_{X}[1,2,3,4]$.

$$
k(n, m)=(x(n), x(m))_{H_{x}}
$$

\section{Definition 1.1}

The random sequence $x(n)$ is called stationary if its covariance function depends only on the difference of its arguments.

$$
k(n, m)=k(n-m)
$$

Contrary to which the sequence $x(n)$ is called nonstationary.

In view of the equality $k(n, m)=\overline{k(m, n)}$, in future we assume that $n \geq m$.

\section{Definition 1.2}

The covariance difference of the sequence $x(n)$ is a function of two natural arguments:

$$
w(n, m)=k(n, m)-k(n+1, m+1)
$$

It is clearly seen that the case when the sequence $x(n)$ is stationary then $w(n, m)=0$ and therefore covariance difference can serve as a measure of deviation of the sequence from its stationariness.

\section{LEMMA 1.1}

Let $x(n)$ and $y(n)$ be two random sequences such that: $(x(n), x(m))=(y(n), y(m))$, then there exists a unitary operator mapping $H_{X}$ into $H_{y}$ and satisfying the condition:

$$
y(n)=U x(n), \mathrm{n}=0,1,2 \ldots
$$

\section{PROOF}

We first define the operator $\mathbf{U}$ on the vectors of the type $\sum_{k=1}^{n} c_{k} x(k)$ in the following way:

$U\left(\sum_{k=1}^{n} c_{k} x(k)\right)=\sum_{k=1}^{n} c_{k} y(k)$.

From the relationship:

$(x(n), x(m))=(y(n), y(m))$, it follows that the operator $\mathbf{U}$ is isometric, and hence it can be continuously transformed into all $H_{X}$ up to unitary operator with the range $H_{y}$, for which $y(n)=U x(n), \mathrm{n}=0,1,2 \ldots$

\section{EXAMPLES}

1. $x(n)=z(n) f_{0}$, where $z(n)$ is a defined sequence and $f_{0}$ is a random value with $M f_{0}=0$, and $M\left|f_{0}\right|^{2}=\sigma_{0}^{2}$, then $k(n, m)=z(n) \overline{z(m)} \sigma_{0}^{2}$
$w(n, m)=(z(n) \overline{z(m)}-z(n+1) \overline{z(m+1)}) \sigma_{0}^{2}$ It is obviously seen that if $z(n) \overline{z(m)}=z(n-m)$, then the sequence $x(n)$ is stationary.

2. Let $x(n)=\lambda^{n} a_{n}(\omega)$, where $\lambda \neq 0$ is a complex number, $a_{n}(\omega)$, is a sequence of random values such that

$M a_{n} \overline{a_{m}}=F(n-m)$, then

$k(n, m)=\lambda^{n} \overline{\lambda^{m}} F(n-m)$ and

$w(n, m)=\left(1-|\lambda|^{2}\right) \lambda^{n} \overline{\lambda^{m}} F(n-m)$.

From here it follows that the sequence $x(n)$ is stationary if and only if $|\lambda|=1$. 
LINEARLY REPRESENTABLE SEQUENCES. THEOREM ON LINEAR REPRESENTABILITY OF A SEQUENCE

\section{Definition 1.3}

The random sequence $x(n)$ is said to be linearly representable, if in $H_{x}$ it has the form:

$x(n)=T^{n} x_{0}(n=1,2 \ldots)$

Where $\mathbf{T}$ is a linear bounded operator acting in $H_{x}, x_{0}$ is a fixed element in $H_{x}$. The spectrum of the operator $\mathbf{T}$ we shall call the spectrum of the sequence $x(n)$. Let $x(n)$ be a stationary sequence and consider the space $H_{x}$ such that the operator $\mathbf{V}$ is acting according to the formulas:

$V x(n)=x(n+1)$

$V\left(\sum_{k=0}^{n} c_{k} x(k)\right)=\sum_{k=0}^{n} c_{k} x(k+1)$

From the stationariness of the sequence $x(n)$, it follows that the operator $\mathbf{V}$ is isometric, Further it is easy to see that

$x(n)=V^{n} x_{0}, x_{0}=x(o)$. Hence therefore any stationary sequence can be represented in the form $x(n)=V^{n} x_{0}, x_{0}=x(o)$, where $\mathbf{V}$ is an isometric operator acting in $H_{x}$.

\section{LEMMA 1.2}

If the sequences $x(n)$ and $y(n)$ have the covariance functions coinciding and one of them is linearly representable, then the other is also linearly representable in Hilbert space.

\section{PROOF}

Let $x(n)$ and $y(n)$ be two random sequences such that: $k_{x}(n, m)=k_{y}(n, m)$ and suppose that $x(n)=T^{n} x_{0}$, where $T \in\left[H_{x}, H_{x}\right], x_{0} \in H_{x}$. From lemma (1.1) it follows that there exists a unitary operator $\mathbf{U}$ mapping
$H_{x}$ onto $H_{Y}$ and satisfying the condition: $y(n)=U x(n) \mathrm{n}=0,1,2 \ldots$ then letting $\hat{T}=U T U^{-1}$ and $y_{0}=U x_{0}$, we arrive at the relationship $y(n)=\widehat{T^{n}} y_{0}, \mathrm{n}=0,1,2 \ldots$ and the lemma is proved.

\section{THEOREM 1.1 (On criteria of linear representability)}

For any given complex-valued function of two arguments $R(n, m)$ to be a covariance function of some linearly representable sequence, it is necessary and sufficient that:

1. $R(n, m)$ be a Hermitian nonnegative i.e.

$\sum_{n, m=0}^{N} R(n, m) \lambda_{n} \overline{\lambda_{m}} \geq 0$ for any sequence of complex numbers $\left\{\lambda_{k}\right\}_{k=1}^{N}$

2. $\left|\sum_{n=0}^{N} \sum_{m=0}^{M} a_{n} \overline{b_{m}} V(n, m)\right|^{2}$

$\leq \mu \sum_{n, \ell=0}^{N} R(n, \ell) a_{n} \overline{a_{\ell}} \cdot \sum_{m, p=0}^{M} R(m, p) b_{m} \overline{b_{p}}$

for any sequence of complex numbers $\left\{a_{n}\right\}_{n=1}^{N}$, $\left\{b_{m}\right\}_{m=1}^{M}$, where $0<\mu<\infty$ and $V(n, m)=$ $R(n+1, m)-R(n, m)$

3. $\quad R(n, m)=\overline{R(m, n)}, \mathrm{n}, \mathrm{m}=0,1,2, \ldots$

\section{Proof}

Assume that there exists a linearly representable sequence $x(n)=T^{n} x_{0}$, such that $k(n, m)=R(n, m)$, then

1. $\sum_{n . m=0}^{N} R(n, m) \lambda_{n} \overline{\lambda_{m}}=\sum_{n, m=0}^{N} k(n, m) \lambda_{n} \overline{\lambda_{m}}=$ 


$$
\begin{aligned}
& \left(\sum_{n=0}^{N} \lambda_{n} x(n) \cdot \sum_{m=0}^{N} \lambda_{m} x(n)\right)=\|g\|^{2} \geq 0 . \text { Where } \\
& g=\sum_{n=0}^{N} \lambda_{n} x(n) .
\end{aligned}
$$

2. $V(n, m)=R(n+1, m)-R(n, m)=$

$k(n+1, m)-k(n . m)=((T-I) x(n), x(m))$.

On the other hand

$$
\begin{aligned}
& \sum_{n, m=0}^{N, M} a_{n} \overline{b_{m}}\left((T-I) T^{n} x_{0}, T^{n} x_{0}\right)= \\
& \left.\left((T-I)\left(\sum_{n=0}^{N} a_{n} T^{n} x_{0}\right), \sum_{m=0}^{M} b_{m} T^{m} x_{0}\right)\right)
\end{aligned}
$$

from where it follows that

$$
\begin{aligned}
& \left|\sum_{n=0}^{N} \sum_{m=0}^{M} a_{n} \overline{b_{m}} v(n, m)\right|^{2} \leq \\
& \|T-I\|^{2} \cdot\left\|\sum_{N=0}^{N} a_{n} T^{n} x_{0}\right\|^{2} \cdot\left\|\sum_{m=0}^{M} b_{m} T^{n} x_{0}\right\|^{2} \text {. Since }
\end{aligned}
$$$$
\left\|\sum_{n=0}^{N} a_{n} T^{n} x_{0}\right\|^{2}=\sum_{n, \ell=0}^{N} R(n, \ell) a_{n} \overline{a_{\ell}} \text {, then finally }
$$$$
\left|\sum_{n=0}^{N} \sum_{m=0}^{M} a_{n} \overline{b_{m}} v(n, m)\right|^{2}
$$$$
\leq \mu \cdot \sum_{n, \ell=0}^{N} R(n, \ell) a_{n} \overline{a_{l}} \cdot \sum_{m, p=0}^{M} R(m, p) b_{m} \overline{b_{p}}
$$

For $\mu$ we can take $\mu=\|T-I\|^{2}$ and hence the proof of the necessary condition is established.

\section{SUFFIENT CONDITION}

Let $\mathbf{E}$ be a Euclidean space, such that $\operatorname{dim} \mathrm{E}=\mathrm{r}$ (the case when $r=\infty$, E is considered to be separable). Let $e_{k}$ for $\mathrm{k}=0,1,2, .$. be an abstract elements. Consider the linear space

$$
\begin{aligned}
& \hat{H}=\left\{\sum_{\ell=0}^{m} e_{\ell} h_{\ell} ; h_{\ell} \in E, m=0,1,2 \ldots\right\} \text { and } \\
& \lambda\left(\sum_{\ell=0}^{m} e_{\ell} h_{\ell}\right)=\sum_{\ell=0}^{m}\left(\lambda h_{\ell}\right)
\end{aligned}
$$

In $\widehat{H}$ we define the bilinear form in the following way:

$$
\left(\sum_{k=0}^{N} e_{k} h_{k}, \sum_{\ell=0}^{M} e_{\ell} \widehat{h_{\ell}}\right)=\sum_{k=0}^{N} \sum_{\ell=0}^{M}\left(R(k, e) h_{k}, \widehat{h_{\ell}}\right)=
$$

$\sum_{k=0}^{N} \sum_{\ell=0}^{M} R(k, \ell) \sum_{\alpha=1}^{n} \lambda^{(k)} \overline{\lambda_{\alpha}^{(\ell)}}$

where

$h_{k}=\sum_{\alpha=1}^{r} \lambda_{\alpha}^{(k)} a_{\alpha}, \widehat{h_{\ell}}=\sum_{\alpha=1}^{r} \overline{\lambda_{\alpha}^{(\ell)}} a_{\alpha}$ and $\left\{a_{\alpha}\right\}_{\alpha=1}^{r}$ is orthonormal bases in $\mathrm{E}$. We show that thus defined bilinear form has all the properties of a scalar product.

a) $\quad\left(e_{k} h_{1}, e_{\ell} h_{2}\right)_{\widehat{H}}=R(k, \ell) \cdot\left(h_{1}, h_{2}\right)_{E}$

$$
\begin{aligned}
& =\overline{R(e, k) \cdot\left(h_{2}, h_{1}\right)_{E}}=\left(e_{\ell} h_{2}, e_{k} h_{1}\right) \text {. } \\
& \text { b) }\left(\sum_{k=0}^{N} e_{k} h_{k}, \sum_{\ell=0}^{N} e_{\ell} h_{\ell}\right)=\sum_{k, \ell=0}^{N} R(k, \ell) \sum_{\alpha=1}^{r} \lambda_{\alpha}^{(k)} \overline{\lambda_{\alpha}^{(\ell)}} \\
& =\sum_{\alpha=1}^{r} \sum_{k, \ell=0}^{N} R(k, \ell) \lambda_{\alpha}^{(k)} \overline{\lambda_{\alpha}^{(\ell)}} \geq 0
\end{aligned}
$$

Homogeneity and linearity properties are easily checked. After closing and factoring according to the nucleus of the bilinear form we obtain Hilbert space $\mathbf{H}$. Fix in $\mathbf{E}$ one element $x_{0}$ such that $\left\|x_{0}\right\|=1$ and consider the sequence $x(n)=e_{n} x_{0}$. Its obvious that

$$
(x(n), x(m))_{H}=R(n, m) \text {. }
$$

Let us denote $H_{0}$ to be a linear closed span of the sequence $\{x(n)\}$ and define a bilinear form $\phi$ in the following way: given that 


$$
\begin{aligned}
& h=\sum_{k=0}^{n} a_{k} x(k) \text { and } \mathrm{g}=\sum_{\mathrm{p}=0}^{\mathrm{m}} \mathrm{b}_{\mathrm{p}} x(p) \text { then } \\
& \phi(h, g)=\sum_{k=0}^{n} \sum_{p=0}^{m} a_{k} \overline{b_{p}} v(k, p) .
\end{aligned}
$$

According to condition 2 of the theorem, the bilinear functional $\phi$ is bounded and consequently according to Riesz's theorem has the form $\phi(h, g)=(A h, g)_{H_{0}}$ where $\mathbf{A}$ is a linear operator acting in $H_{0}$.

Let $g=\sum_{p=0}^{m} b_{p} x(p)$, then

$$
(x(n+1), g)-(x(n), g)=
$$$$
\sum_{p=0}^{m} \overline{b_{p}}[(x(n+1)-x(n), x(p))]=
$$$$
\sum_{p=0}^{m} \overline{b_{p}} v(n, p)=\phi(x(n), g)=(A x(n), g) \cdot \text { Since }
$$

the set of vectors of the type $\sum_{p=0}^{m} b_{p} x(p)_{\text {is complete in }}$ H, then $x(n+1)=(A+I) x(n)$. If we set $T=(A+I)$, we obtain $x(n)=T^{n} x_{0}, x_{0}=x(0)$ i.e. $e_{0}=1$ and $R(n, m)=K(n, m)$ and the theorem is proved,

\section{ONQUASISTATIONARITY}

Let $x(n)$ be a random sequence. Consider the quadratic form

$$
\sum_{\alpha, \beta=0}^{m} w(\alpha, \beta) \lambda_{\alpha} \overline{\lambda_{\beta}},\left(\lambda_{\alpha} \in \mathbb{C}, m=0,1,2 \ldots\right)
$$

The sequence $x(n)$ is called quasistationary, if the rank of the quadratic form (1.5) is bounded and the maximum rank $\rho$ is called the rank of nonstationariness. Its obvious that, if the sequence $\mathrm{x}(\mathrm{n})$ is stationary, then $\rho=0$ and therefore the rank of nonstationariness characterizes the degree of deviation of the sequence from its stationariness.

Let there be given a linearly representable sequence

$x(n)=T^{n} x_{o}$. It is clear that

$$
w(\alpha, \beta)=\left(\left(I-T^{*} T\right) x(\alpha), x(\beta)\right)=
$$

$$
\sum_{j=1}^{r}\left(x(\alpha), g_{j}\right) \cdot\left(g_{j}, x(\beta)\right)
$$

where $r=\operatorname{dim}\left(I-T^{*} T\right) H, I-T^{*} T=\sum_{J=1}^{r}\left\langle., g_{j}\right\rangle g_{j}$ and therefore

$$
\begin{aligned}
& \sum_{\alpha, \beta=0}^{m} w(\alpha, \beta) \lambda_{\alpha} \overline{\lambda_{\beta}} \\
& =\sum_{\alpha, \beta=0}^{m} \sum_{j=1}^{r}\left(\lambda_{\alpha} x(\alpha), g_{j}\right) \cdot\left(g_{\alpha}, \lambda_{\beta} x(\beta)\right) \\
& =\sum_{j=1}^{r}\left|\left(\sum_{\alpha=0}^{m} \lambda_{\alpha} x(\alpha), g_{j}\right)\right|^{2}
\end{aligned}
$$

from where for any $m=0,1,2 \ldots$ The rank of the quadratic form (1.5) does not exceed $\mathrm{r}$.

\section{THEOREM1.2.(ONRANK)}

In order for a linearly representable sequence to be quasistationary of rank $\rho$, it is necessary and sufficient that $\operatorname{dim}\left(I-T^{*} T\right) H=r<\infty$, in which case $\rho=r$. PROOF

It is enough in the proof to establish only the necessary condition.

For any $\mathrm{m}=0,1,2 \ldots$ we let $\mathrm{H}(\mathrm{m})$ to be a subspace consisting of the elements of the type:

$h=\sum_{k=0}^{m} c_{k} x(k), c_{k} \in \mathbb{C}$, then 


$$
\left(\left(I-T^{*} T\right) h, h\right)=\sum_{\alpha, \beta=0}^{m} w(\alpha, \beta) \lambda_{\alpha} \overline{\lambda_{\beta}},(\lambda \in H(m))
$$

Define the subspace $G_{m}=P_{m}\left(I-T^{*} T\right) H$, where $p_{m}$ is the orthornormal projection on $H(m)$. Its clear that $G_{m} \subseteq p_{m}\left(I-T^{*} T\right) H$. From (1.6) it follows that the rank of the quadratic form

$$
\sum_{\alpha, \beta=0}^{m} w(\alpha, \beta) \lambda_{\alpha} \overline{\lambda_{\beta}},\left(\left(I-T^{*} T\right) h, h\right),
$$

$\left(h=\sum_{\alpha=0}^{m} \lambda_{\alpha} x(\alpha)\right)$ equals $\operatorname{dim} G_{m}$. Consider the sequence $H(1) \subset H(2) \subset \ldots$... Since the sequence $\mathrm{x}(\mathrm{n})$ is embedded in the space $H=H_{X}$ then $\lim _{m \rightarrow \infty} p_{m}=I$ and consequently

$\lim _{m \rightarrow \infty} G_{m}=\left(I-T^{*} T\right) H$ from the relationship

$\operatorname{dim} G_{m} \leq \rho,(\mathrm{m}=0,1,2 \ldots)$ it follows that

$r=\operatorname{dim}\left(I-T^{*} T\right) H=\lim _{m \rightarrow \infty} \operatorname{dim} G_{m} \leq \rho$

since $\rho \leq r$ then $r=\rho$ and the theorem is proved.

\section{DISSIPATIVE RANDOMSEQUENCES}

\section{Definition 1.4}

A quasistationary sequence $\mathrm{x}(\mathrm{n})$ is called dissipative if nonnegative all the quadratic forms:

$$
\sum_{n . m=0}^{N} w(n, m) \lambda_{n} \overline{\lambda_{m}} \geq 0
$$

From the definition it follows that, if a random sequence is dissipative, then the quadratic form

$$
\sum_{n . m=0}^{N} k(n+p, m+p) \lambda_{n} \overline{\lambda_{m}} \text { is a nonincreasing }
$$

sequences in $\mathrm{p}$.
In particular, the sequence $\mathrm{k}(\mathrm{n}, \mathrm{n})$ is nonincreasing in $\mathrm{n}$. From here it follows that there exists the limit:

$$
\lim _{n \rightarrow \infty} k(n, n)=\sigma_{\infty}^{2}
$$

Two cases are possible here:

1. $\sigma_{\infty}^{2}=0$

2. $\sigma_{\infty}^{2}>0$

In the first case, we say that the sequence $\mathrm{x}(\mathrm{n})$ is asymptotically diminishing while in the second case, we say that the sequence is asymptotically nondiminishing.

\section{LEMMA 1.3}

For dissipative sequences, there always exist the limit:

$$
\lim _{p \rightarrow \infty} k(n+p, m+p)
$$

\section{PROOF}

Consider the relationship

$$
k(n, m)=\frac{1}{4}\left\{\begin{array}{l}
\|x(n)+x(m)\|^{2}+\|x(n)-x(m)\|^{2} \\
+i\|x(n)+i x(m)\|^{2}-i\|x(n)-i x(n)\|^{2}
\end{array}\right\}
$$

Since

$$
\left\|\sum_{k=0}^{n} \lambda_{k} x(k+p)\right\|^{2}=\sum_{k, q=0}^{N} k(k+p, q+p) \lambda_{k} \overline{\lambda_{q}},
$$

then for a dissipative sequence the quadratic form $\left\|\sum_{k=0}^{N} \lambda_{k} x(k+p)\right\|^{2}$ is nonincreasing in $\mathrm{p}$.

If we change in (1.8) $\mathrm{n}$ with $\mathrm{n}+\mathrm{p}$ and $\mathrm{m}$ with $\mathrm{m}+\mathrm{p}$, we obtain that every summand in (1.8) has a limit and consequently there exist the limit:

$$
\lim _{p \rightarrow \infty} k(n+p, m+p) \text {. Let } x(n)=T^{n} x_{0} \text { be a linearly }
$$

representable sequence. Since in this case, the covariance difference has the form

$$
w(n, m)=\left(\left(I-T^{*} T\right) x(n), x(m)\right)=
$$




$$
\sum_{\alpha=1}^{r}\left(x(n), g_{\alpha}\right) \cdot\left(g_{\alpha,} x(n)\right)
$$

where, $r=\operatorname{dim}\left(I-T^{*} T\right) H,\left(I-T^{*} T\right)=\sum_{\alpha=1}^{r}<., g_{\alpha}>g_{\alpha}$, then it is easy to note that if $\mathbf{T}$ is a contraction operator, then the sequence $\mathrm{x}(\mathrm{n})$ is dissipative. The next theorem proves that, among the linearly representable sequences, dissipative are only those sequences whereby in their representations $\mathbf{T}$ is a contraction operator.

\section{THEOREM 1.3}

In order for a linearly representable sequence $x(n)=T^{n} x_{0}$, to be dissipative, it is necessary and sufficient that $\mathbf{T}$ be a contraction operator and $\operatorname{dim}\left(I-T^{*} T\right) H<\infty$.

\section{Proof}

It remains to prove only the necessary condition. Let $x(n)=T^{n} x_{0}$, be a dissipative sequence, then according to the definition it is quasistationary and in view of theorem (1.2), $\operatorname{dim}\left(I-T^{*} T\right) H=r<\infty$. On the other hand from the relationship (1.7) it follows that: $\left(\left(I-T^{*} T\right) h, h\right) \geq 0, h=\sum_{\alpha=0}^{m} \lambda_{\alpha} x(\alpha)$. Since the vectors of the type $\sum_{\alpha=0}^{m} \lambda_{\alpha} x(\alpha)$ are dense in $H_{x}$, then the operator $\mathbf{T}$ is bounded and consequently for any $h \in H_{x},\left(\left(I-T^{*} T\right) h, h\right) \geq 0$.

Let $\mathbf{T}$ be a contraction operator acting in Hilbert space $\mathbf{H}$. It is clear that the sequence $A_{n}=T^{*^{n}} T^{n}$ is a monotonic nondecreasing sequence of positive bounded operators. According to the theorem on convergence of the sequences of operators [5], there exists a strong limit: $R=s . \lim _{n \rightarrow \infty} T^{*^{n}} T$. It is easy to note that $\mathbf{R}$ is a positive contraction operator satisfying the relationships:

$$
\begin{array}{ll}
\text { 1. } & T^{*^{m}} R T^{n}=R T^{n-m},(n \geq m) \\
\text { 2. } & \lim _{p \rightarrow 0} k(n+p, m+p)=\left(R T^{n-m} x_{0}, x_{0}\right)=k_{\infty}(n-m) \\
\text { 3. } & \lim _{p \rightarrow \infty} w(n+p, m+p)=0
\end{array}
$$

\section{THEOREM 1.4}

Let $\mathrm{T}$ be a contraction operator acting in $\mathrm{H}$. Suppose that $\left\{T^{*^{n}}\left(I-T^{*} T\right) H\right\}=H$, then the sequence $G_{n}=T^{n}$, $\mathrm{n}=0,1,2 \ldots$ tend to Zero in a weaker sense when $n \rightarrow \infty$.

\section{PROOF}

Since the operator $\mathrm{T}$ is a contraction operator, then there exists an operator

$$
\left(I-T^{*} T\right)^{\frac{1}{2}}, \text { Such that for any } h \in H
$$

$\lim _{n \rightarrow \infty}\left\|\left(I-T^{*} T\right)^{\frac{1}{2}} T^{n} h\right\|^{2}=\lim _{n \rightarrow \infty}\left(\left(I-T^{*} T\right) T^{n} h, T^{n} h\right)=0$

Let $g \in H$ and $k \in \mathbb{Z}^{+}$, then

$\lim _{n \rightarrow \infty}\left(T^{n} h, T^{*^{k}}\left(I-T^{*} T\right) g\right)=$

$\lim _{n \rightarrow \infty}\left(\left(I-T^{*} T\right) T^{n+k} h, g\right)=0$

From here and also the comparison $\left\|T^{n}\right\| \leq 1, \mathrm{n}=0,1,2, \ldots$ follows that the sequence $G_{n}=T^{n}$ tends to zero in a weak sense when $n \rightarrow \infty$

\section{CLASSIFICATIONS OF DISSIPATIVE LINEARLY REPRESENTABLESEQUENCES}

Let in Hilbert space $\mathbf{H}$ be given a contraction operator $\mathbf{T}$, such that there exist a complex number $\mathrm{r}|r|=1$ and that the operator $(r I-T)$ is invertible in a weaker sense i.e. 
the operator $(r I-T)^{-1}$ is bounded and everywhere defined. Consider $T^{\prime}=\bar{r} T$, it is possible without loss of generality to consider that $r=1$. If therefore $\mathbf{T}$ is a contraction operator in $\mathbf{H}$ such that the point 1 is a regular point for it, then consider the operator

$$
A=i(I+T)(I-T)^{-1}=2 i(I-T)^{-1}-I
$$

The operator A thus defined is called Kelley's transformation of the operator T. From an obvious relationship

$$
\frac{A-A^{*}}{i}=2\left(I-T^{*}\right)^{-1}\left(I-T^{*} T\right)(I-T)^{-1}
$$

follows that the operator $\mathbf{A}$ is a dissipative bounded operator of Hermitian rank equal to rank of nonunitary operator $\mathbf{T}$. The operator $\mathbf{T}$ is defined through the operator A with the aid of the formulas:

$$
T=(A-i I)(A+i I)^{-1}=I-2 i(A+i I)^{-1}
$$

In future we consider only quasiunitary contraction operators for which

$$
\operatorname{dim}\left(I-T^{*} T\right) H=\operatorname{dim}\left(I-T T^{*}\right) H=\rho<\infty
$$

We state without proof some necessary information for future purposes regarding Kelley's transformations.

\section{Theorem 1.5}

The operators $\mathbf{T}$ and $\mathbf{A}$ have similar invariant subspaces, whereas if $H_{0}$ is invariant in relation to A subspace, then:

$$
T /_{H_{0}}=\left(A /_{H_{0}}-i I I_{H_{0}}\right)\left(A /_{H_{0}}+i I I_{H_{0}}\right)^{-1}
$$

\section{Theorem 1.6}

The operator $\mathbf{T}$ in nonunitary(simple) if and only if the operator $\mathbf{A}$ is non-selfadjoint(simple).

\section{Proof}

Assume that the operator $\mathbf{T}$ is simple. If the operator

$$
A=i(I+T)(I-T)^{-1}
$$

is not simple, then the space $\mathbf{H}$ is a direct sum of two invariant in relation to $\mathbf{A}$ subspaces $H_{0}$ and $H_{1}$, such that the operator $A_{0}=A /_{H_{0}}$ is self-adjoint operator [7].
From theorem (1.5) it follows that $H_{0}$ and $H_{1}$, are invariants in relation to $\mathbf{T}$ and $T_{0}=T /_{H_{0}}=\left(A_{0}-i I_{H_{0}}\right)$ $\left(A_{0}+i I_{H_{0}}\right)^{-1}$ then from obvious relationships

$$
\begin{aligned}
& \left(I-T^{*} T\right)=2\left(A^{*}-i I\right)^{-1}\left(\frac{A-A^{*}}{i}\right)(A+i I)^{-1} \\
& \left(I-T T^{*}\right)=2(A+i I)^{-1}\left(\frac{A-A^{*}}{i}\right)\left(A^{*}-i I\right)^{-1}
\end{aligned}
$$

it follows that $T_{0}=T / H_{0}$ is a unitary operator which contradicts initial assumption. The sufficient condition is established analogously.

\section{Theorem 1.7}

Let $A_{1}$ and $A_{2}$ be two dissipative bounded operators acting in $H_{1}$ and $H_{2}$ correspondingly. Let $T_{1}$ and $T_{2}$ be their corresponding Kelley's transformations. Then if in a certain invariant subspace $H_{0} \subset H_{1}$, the operator $A_{1}$ is unitary equivalent to operator $A_{2}$, then in the same subspace, the operator $T_{1}$ is unitary equivalent to the operator $T_{2}$.

The proof of this theorem follows from (1.11)

The importance of the last theorem is that it helps to construct the universal models of quasiunitary contraction operators with the aid of universal models of dissipative operators $[3,7,8]$.

\section{Definition 1.5}

The dissipative operator $\mathbf{A}$ is called complete, if the linear closed span of all its invariants subspaces, corresponding to nonreal eigen values of the spectrum (points of the spectrum lying inside a unit circle) coincides with $\mathrm{H}$.

Let $\mathbf{T}$ be a contraction operator in $\mathbf{H}$, such that (I-T) is invertible in a simple sense and let

$$
A=i(I+T)(I-T)^{-1} \text {. From the relationships: }
$$




$$
\begin{aligned}
& (I-\lambda I)^{-1}=\frac{1}{1-\lambda}(A+i I)\left[A-i \frac{1+\lambda}{1-\lambda}\right], \lambda \neq 1 \\
& (A-z I)^{-1}=\frac{1}{z+i}(I-T)\left[T-\frac{z-i}{z+i}\right],(z \neq-i)
\end{aligned}
$$

follows that the point $\lambda \neq 1 \lambda \neq 1$ belongs to the spectrum of the operator $\mathbf{T}$ if and only if, when the point $z=\frac{i(1+\lambda)}{1-\lambda}$ belongs to the spectrum of the operator $\mathbf{A}$ and is an easy matter to prove that the real vector $\mathbf{h}$ of the operator $\mathbf{T}$ corresponds to the real number $\lambda$ if and only if when $g=(A+i I)^{-1} h$ is a real vector of operator $\mathbf{A}$ and corresponds to the real number

$$
z=\frac{i(1+\lambda)}{1-\lambda}
$$

\section{Theorem 1.8}

The operator $\mathbf{T}$ is complete if and only if when $A=i(I+T)(I-T)^{-1}$ is complete.

\section{Theorem 1.9}

Let $\mathbf{T}$ be a contraction quasiunitary operator of rank $\rho<\infty$, such that the operator (I-T) is invertible in a simple sense. Then any point of the spectrum of the operator $\mathbf{T}$, lying inside a unit circle is a real eigen value of a finite multiplicity.

\section{Proof}

Consider the operator $A=i(I+T)(I-T)^{-1}$. From the finiteness of the subspace $\left(I-T^{*} T\right) H$, it follows that the operator

$$
\frac{A-A^{*}}{i}=2\left(I-T^{*}\right)^{-1}\left(I-T^{*} T\right)(I-T)^{-1} \text { is completely }
$$

continuous.
Let now $\lambda(|\lambda|<1)$ be the point of the operator $\mathbf{T}$, then the point $z=\frac{i(1+\lambda)}{1-\lambda}$ belongs to the spectrum of the operator A and consequently in view of the theorem 5.2 [9], $\mathrm{z}$ is a nonreal eigen value of the operator $\mathrm{A}$ of finite multiplicity.

The theorem 1.9 was derived in the works [9] for a more wider class of operators, that allows a representation of the form: $\mathrm{T}=\mathrm{U}+\mathrm{B}$

Where $\mathbf{U}$ is a unitary operator and $\mathbf{B}$ is a completely continuous operator with a view that the operator $\mathbf{T}$ has one regular point inside the unit circle. Let now be given a random linearly representable sequence $x(n)=T^{n} x_{0}$, where $\mathbf{T}$ is a contraction quasiunitary operator and the operator (I-T) is invertible in a simple sense. As was mentioned earlier the operator $A=i(I+T)(I-T)^{-1}$ is bounded and dissipative operator of a finite nonHermitian rank. Its known $[7,10]$ that such operators allows the representation of the form:

$$
\operatorname{det} w_{A}(\lambda)=\prod_{j=1}^{\infty} \frac{\overline{z_{j}}-\lambda}{z_{j}-\lambda} e^{i_{0}^{\ell} \frac{d t}{\lambda-\alpha(t)}},(\ell>0)
$$

where $\alpha(x)$ is a nonincreasing function on $[0, \ell]$ and $\left\{z_{k}\right\}$ is a sequence of nonreal eigen values of the operator A.

$$
w_{A}(\lambda)=I-i\left\|(A-\lambda I)^{-1} g_{\alpha}, g_{\beta}\right\|
$$

$\frac{A-A^{*}}{i}=\sum_{\alpha=1}^{r}<., g_{\alpha}>g_{\alpha}$ and $r=\operatorname{dim}(2 \operatorname{Im} A H)$.

Using the fact that $z_{j}=i \frac{1+\lambda_{j}}{1-\lambda_{j}}, \mathrm{j}=1,2,3 \ldots$ Where $\left\{\lambda_{j}\right\}_{j=1}^{\infty}$ is a sequence of eigen values of the operator $\mathbf{T}$, lying inside the unit circle, we obtain 
$\operatorname{det} w_{A}(\lambda)=\prod_{j=1}^{\infty} \frac{\overline{\lambda_{j}}(\lambda-i)-(1+\lambda)}{\lambda_{j}(\lambda+i)+(i-\lambda)} \times \frac{1-\lambda_{\mathrm{j}}}{1-\bar{\lambda}_{\mathrm{j}}} e^{i_{0}^{\ell} \frac{d t}{\lambda-\alpha(t)}}$

We are going to say that a dissipative linearly representable sequence

$x(n)=T^{n} x_{0}$ belongs to the class $H^{(r)}\left[\lambda_{k}, \alpha(x)\right]$,

$\mathrm{r}<\infty$ if the rank of the quasiunitary operator does not

exceed $\mathrm{r}(\rho \leq r)$.

The operator (I-T) is invertible in a simple sense and the operator $A=i(I+T)(I-T)^{-1}$ is true for the relationship (1.15). If the discrete operator is missing, then the corresponding class we denote by $H^{(r)}\left[\lambda_{k}\right]$. In case of a complete operator $\mathrm{T}$, the corresponding class we denote by $k^{(r)}\left[\lambda_{k}\right]$.

\section{CONCLUSION}

From the first chapter it follows that for linearly representable sequences $x(n)=T^{n} x_{0}$, the properties of the quasistationarity and dissipativeness are only expressed through the properties of the operator $\mathbf{T}$. Further, for some bounded on the operator $\mathbf{T}$, theorem 1.9 allows to obtain the classifications of such sequences only according to the spectrum of the operator $\mathbf{T}$.

\section{REFERENCES}

1. A.N. Kolmogorov. Stationary sequences in Hilbert spaces. (1941), pp. 1-40.

2. A.M. Yagrom. Introduction to the theory of stationary random functions. (1952), pp.3-168.

3. M.C. Livshit, A.A. Yantsevich. Operator colligations in Hilbert spaces. (1971), pp. 5-160.

4. A.A. Yantsevich. Nonstationary sequences in Hilbert spaces. (1986), pp. 139-141.

5. N.I. Akhiezer, I.M. Glazman. Theory of linear operators in Hilbert space. (1966), pp.20-54.

6. B. Nagia, K. Foyash. Harmonic analysis of operators in Hilbert space. (1970),pp. 20-27.

7. M.S.Brodskii. Triangular and Jordans representations of linear operators. (1969), pp. 287300.

8. K.P. Kirchev. About one class o nonstationary random processes. (1971), pp.150-169.

9. M.F.Krein. Introduction to the theory of linear nonself adjoint operators. (1965), pp.15-20.

10. M.S. Brodskii, M.S. Livshit. Spectral theory of nonself adjoint operators and middle systems. (1958),pp. 385. 
